
for Mechanical Engineering

\title{
Numerical study of Flow patterns and performance of a coupled cavity-dish system under different focal lengths
}

\begin{tabular}{|r|l|}
\hline Journal: & Transactions of the Canadian Society for Mechanical Engineering \\
\hline Manuscript ID & TCSME-2021-0110 \\
\hline Manuscript Type: & Article \\
\hline Date Submitted by the & $08-J u l-2021$ \\
\hline $\begin{array}{r}\text { Complete List of Authors: } \\
\text { Azair, Muhammad; NED University of Engineering \& Technology, } \\
\text { Department of Mechanical Engineering } \\
\text { Siddiqui, Mubashir; NED University of Engineering \& Technology, } \\
\text { Department of Mechanical Engineering } \\
\text { Allauddin, Usman; NED University of Engineering \& Technology, } \\
\text { Department of Mechanical Engineering }\end{array}$ & $\begin{array}{l}\text { Coupled cavity-dish system, focal lengths, wind flow, wind loading, } \\
\text { convective heat loss }\end{array}$ \\
\hline $\begin{array}{r}\text { Keywords: the invited manuscript for } \\
\text { consideration in a Special } \\
\text { Issue? : }\end{array}$ & \begin{tabular}{l} 
Not applicable (regular submission) \\
\hline
\end{tabular} \\
\hline
\end{tabular}

\section{SCHOLARONE \\ Manuscripts}




\title{
Numerical study of Flow patterns and performance of a coupled
}

\author{
cavity-dish system under different focal lengths \\ Muhammad Uzair ${ }^{*}$, Mubashir Ali Siddiqui ${ }^{1}$, Usman Allauddin ${ }^{1}$ \\ ${ }^{1}$ Department of Mechanical Engineering, NED University of Technology, Pakistan \\ *Corresponding author: uzair@neduet.edu.pk
}

\begin{abstract}
:
The effectiveness of the parabolic dish system (PDS) is greatly affected by the heat losses associated with high temperatures. The complexity of flow and temperature patterns in and around the cavity receiver makes it a challenging task to determine the convective heat loss from the cavity. Various studies have been carried out to determine the convection heat losses from isolated cavities of different shapes. In the presence of dish structure, the free stream wind may affect the stability of structure and the heat losses from the PDS. In this study, effect of focal length on the performance of the coupled cavity-dish system was analyzed using numerical simulations. The loading and the convective heat loss from the cavity were examined with three different cavity positions and different operating conditions in the presence of the dish. The results showed that the shallow dish experienced higher local air velocities near the cavity receiver than in the case of the deep dish. It was concluded that the heat loss is a stronger function of tilt angle rather than focal length, and in essence, the heat losses due to variation of this are negligible.
\end{abstract}

Keywords: Coupled cavity-dish system; focal lengths; wind flow; wind loading; convective heat loss 


\section{Introduction:}

Due to high demand and increase in the energy price for residential and industrial applications, the use of alternate renewable energies, especially solar energy, has tremendously increased in past few years. Solar thermal power is the one of the most cost effective techniques for large scale electricity production. Globally, power generation using CSP plants has grown intensely using all four concentrated solar power conversion techniques, and the installed capacity of CSP plants has increased from $4 \mathrm{GW}$ in 2010 to an expected forecast ranging from $30 \mathrm{GW}$ to $40 \mathrm{GW}$ after 2030 (Philibert, 2014). Among all the available concentrated solar power (CSP) system, the parabolic dish collectors are most efficient ones having concentration ratio of more than 1300 Suns compared with parabolic troughs and solar power towers having concentrations of 70-80 Suns and 1000 Suns respectively (Steinfeld, 2005; Tyner et al. 2001). Due to higher temperatures achieved in the cavity, the parabolic dish systems cover a wide range of applications (Wang and Siddiqui, 2010). In general CSPs are located in open terrain and are sensitive to wind. The surrounding air can affect the aerodynamic loads as well as heat loss from the cavity receiver to the ambient air (Lupfert, 2001). In order to analyse the performance of the parabolic dish system, it is required to understand the aerodynamic loading around the solar receiver and the heat transfer from the cavity receiver to the ambient air.

In the design of solar parabolic dish collectors, wind loading is a critical design parameter. Given the importance of accurately designing and predicting the performance of CSP, numerous models have been proposed to evaluate their aerodynamic, optical and thermal performance (Danish et al., 2021; Carrillo-Berdugo et al., 2021; Giaconia et al., 2020; Li et al., 2021; Yang et al., 2021). In many investigations, the wind effects were investigated in term of wind-load coefficients by wind tunnel testing. However, most of these experimental studies were performed on parabolic trough systems to investigate the aerodynamic loads. 
The extensive researches, performed on the wind loading around the structure, have shown the superior performance for the shallow reflectors compared to deep reflectors. The aerodynamics forces increases with increase in the curvature of reflector. As a result, deep reflector experiences more aerodynamics loading than shallow one due to increased curvature.

The performance of solar power plant is significantly influenced by the heat losses from the cavity receiver (Price et al., 2002). For parabolic dish concentrators, the cavity protects the absorbing material placed in it from wind and naturally driven air currents. The cavity position, internal temperatures, along with the wind conditions including both the speed and the direction were investigated and found to significantly affect the rate of heat loss from the cavity receiver (Paitoonsurikaran and Lovegrove, 2003). As expected, the findings showed that the heat loss from thermal receiver significantly reduces the efficiency and accordingly increases the cost value of the system. Due to the importance of thermal losses from the receiver, both experimental and numerical studies on convective heat transfer in receiver have been research areas for researchers, and numerous models have been proposed. A state of the art review by $\mathrm{Wu}$ et al. (2010) covered the studies conducted to examine the convective heat loss from the cavity receiver in detail. Loni et al. (2020) compared the performance of different cavity shapes (cubical, cylindrical, and hemispherical) was performed using pure oil and nano-fluid. They observed an enhancement in the thermal efficiency using hemispherical cavity with nano-fluid. A new indirect-irradiated cavity receiver design was proposed (Sedighi et al., 2020), and the result revealed highest efficiency using cylindrical cavity with an inverted conical base. Alipourtarzanagh (2020) investigated the effect of air curtian on the heat loss upto $60 \%$ from the cavity receiver. Another experimental study was performed on three different cylindrical cavities to develop a corrleation of natural convection heat loss (Abbasi-Shavazi et al., 2020). Yuan et al. (2020) 
also performed experiments to improve the performance of the cavity recevier. They reported an increase in the optical and thermal efficiency. Xiao et al. (2020) perofmed numerical investigation to examin the optical and thermal efficiencies of cylindrical cavity. They analyzed differnet parameters including the rim angle, mass flow rate, all absorpitivity and cavity tilt angle. Pavlovic et al. (2017) performed expermental invetigation to analyse the thermal and exergetic efficiencies of PDS. They used differnet working fluids with differnet combination of temperature and volume flow rate. Surprisingly, numerous other studies also estimated the natural and forced convection heat losses (Reddy et al., 2015, 2016; Thirunavukkarasu and Cheralathan, 2020; Alnaqi et al., 2021; Mehrpooya, 2019), however, most of the studies were performed to analyse the heat loss from isolated cavity receiver decoupled from the dish-cavity system.

In all the works, no cognisance was given to the effect of the position of the focal length of the cavity on the performance of the PDS associated with the convective heat loss and wind loading on the dish structure. The cavity receiver in a parabolic dish system is placed at the focal point of the dish to absorb all the incidence radiations to get a significant higher temperature. The positioning of cavity receiver may affect the structural loading and heat losses from the receiver, and hence overall efficiency of the system. This work aimed to study the impact of different focal point positions on the wind loading and the heat loss from the cavity receiver.

\section{Methodology}

In order to perform a numerical simulation, a dish installed at Australian National University (ANU), having a $5 \mathrm{~m}$ diameter with a reflecting surface of $20 \mathrm{~m}^{2}$, was selected as a reference model. The dish dimensions (Uzair et al., 2017, 2018) are shown in Figure 1a. Using the dish as reference, two more dish geometries were analysed. The aperture area of these three dish 
geometries were kept constant. The focal lengths for these three dish structures were selected as $1.25 \mathrm{~m}$ (deep dish), $1.84 \mathrm{~m}$ (medium dish) and $2.5 \mathrm{~m}$ (shallow dish), where the dish diameter was $5 \mathrm{~m}$. Having a constant diameter and different focal lengths, the rim angles also varied in all cases. The placement of focal point also describes the rim angle. Based on the variation of rim angles, the dish structure can be define as either deep dish or shallow dish. Negligible radiation losses were reported by Wang and Siddiqui (2010) by changing the rim angle of parabolic dish. So, by using deep dish may provide more sheltering effect to dish as compared to shallow dish, and can increase the thermal performance by reducing heat loss. On the other hand, the aerodynamic forces on dish will increase due to increase in the depth of structure. At higher temperature, the saving could be significant by compromising on the aerodynamics forces.

Besides from the fact that the positioning of receiver may affect the heat loss from the receiver, it also changes the concentration ratio of the system. To achieve higher operating temperatures, larger dish structures are used to increase the concentration ratio. The concentration ratio depends on collector geometry, geometrical mirror quality and the mirror reflectivity. Different collector geometries generate different concentration ratios, and among all different shapes, paraboloid shape creates possible the highest concentration ratio. Geometrical mirror quality also plays an important role to improve the concentration ratio. Geometrical errors including slope error of mirror provoke radiation deviation and subsequently effects on the concentration ratio. Mirror reflectivity also effects the concentration ratio, as higher the reflectivity lower will be the absorption and transmission losses at the mirror. At high concentration ratio, higher temperature can be achieve, but increasing the concentration ratio higher than the 2000 , higher thermal losses occurs at higher temperatures and thus no significant increase in the system efficiency. By considering this aspect as well as the expected higher construction cost to achieve higher concentration ratio, 
it may not be economically feasible to increase the concentration ratio over 2000 (Mohr et al., 1999). The size of cavity receiver may also affect the convective heat loss from the receiver. As the receiver opening increases, due to increase in air movement, there could be an increase in the heat losses from the cavity receiver. The increase of the cavity opening may alter the stagnation zone and amount of sweeping the hot air from the receiver due to the wind.

The main purpose of the study was to examine the effect of the cavity's positioning on the wind loading and convective heat loss in the presence of the dish structure. Three dimensional steady state numerical simulations were performed using ANSYS CFX with a free stream velocity of $5 \mathrm{~m} / \mathrm{s}$ and ambient temperature at $25^{\circ} \mathrm{C}$. The Reynold number was found to be $1.9 \times 10^{6}$ using characteristic length as the dimeter of the dish. The Shear Stress Transport (SST) two-equation eddy-viscosity turbulence model was used as it is one of the most accurate two-equation models for separation prediction. In order to examine the flow characteristics, the selection of domain was carried out on different domain sizes and the selected domain was extended $75 \mathrm{~m}$ upstream, $105 \mathrm{~m}$ downstream and $30 \mathrm{~m}$ on lateral direction with respect to the dish structure. The boundary conditions at the domain were selected as noslip condition as there was no effect of walls was experienced on the flow near dish. After careful mesh sensitivity test, a refined mesh of about 2 million was selected. The mesh was well refined near the dish region and scattered in the region away from the dish. The y-plus value near the dish and cavity wall was set to be 1 . The walls of cavity receiver were selected as isotheral walls at $600^{\circ} \mathrm{C}$. The study was conducted numerically and the simulations were performed for different tilt angles ranging from $90^{\circ}$ (when wind approached directly to the face of the dish structure) to $-90^{\circ}$ (when wind approached to back side of the dish structure). The orientation of the dish is shown in Figure $1 \mathrm{~b}$. 


\section{Results and Discussion}

\subsection{Flow pattern}

The velocity contours along the centre plane for different dish tilt angles are shown in Figures 2-10. The flow direction is set from left to right. The local flow near the cavity receiver showed different behaviour depending upon the dish orientation. For tilt angle, the local velocity near the receiver was found to be normal to the free steam flow at the edge of the dish. As a result, the stagnation condition was generated in front of the dish and the velocity values increased at edge of the dish to maintain the continuity. Also the two strong vortices were formed in the wake region of the dish. However, due to positioning of the cavity in three different cases, due to sheltering effects of the deep dish on the receiver, the local velocity near the receiver was lower than other (Figure 2).

Moving to $60^{\circ}$ tilt angle, again two large vortices were generated behind the dish due to the flow separation at the dish edge. However, similar to $90^{\circ}$ tilt angle, the deep dish created the strong stagnation zone near the receiver mouth compared to the other cases (Figure 3). Figures 4 and 5 show the velocity contours for $45^{\circ}$ and $30^{\circ}$ tilt angle. It is evident that the effect of the dish structure on the local flow near the receiver reduces with a reduction in tilt angles. For $30^{\circ}$ tile angle, the local flow was observed to be uniform in all three cases, and there was a minimal variation in the velocity for different cavity positions. For $0^{\circ}$ tilt angle (Figure 6), the medium and shallow dish structures did not show any noticeable effect on the local flow near the cavity receiver. Under these conditions, depending upon the position of the cavity, minimal dish affect was observed on the local flow. However, the local flow was significantly affected in case of the deep dish structure due to the positioning of the receiver (Figure 6a). In this case, the receiver was located within the boundary layer of the dish. 
With the flow from the back side of the dish i.e. negative tilt angles, deep dish again provided more shelter to the receiver and the local velocities were less compared with other cases. Figures 7 and 8 show the velocity contours for $-30^{\circ}$ and $-45^{\circ}$ tilt angles. For $-30^{\circ}$ tilt angle, owing to the positioning of the receiver in the wake region of the dish, the receiver experienced a vortex due to flow from the back side of the dish (Figure 7). Similarly, a large recirculation was observed for $-45^{\circ}$ tilt angle (Figure 8 ). However, the local velocities near the receivers were high for shallow dish.

By increasing the tilt angles to $-60^{\circ}$ and $-90^{\circ}$ (Figure 9-10), high velocities were observed at the edges of the dish structure. Due to these high velocities, negative pressure was observed in the wake region that ultimately generated strong vortices for $-90^{\circ}$ tilt angle (Figure 10).

By observing all the cases, it can be noted that the shallow dish experienced higher local air velocities near the cavity receiver than in the case of the deep dish. In most of the cases, the local air near the receiver was not significantly affected by the dish compared to deep dish.

\subsection{Drag Coefficients}

In the design of solar parabolic dish collectors, wind loading is one of the major design parameter to consider critically. The geometry of the dish also plays a key role to investigate the aerodynamic behaviour on and around the dish. The extensive research, performed on the wind loading around the structure, have shown the superior performance for the shallow reflectors compared to deep reflectors. The aerodynamics forces increased with increase in the curvature of reflector. As a result, deep reflector experienced more aerodynamics loading than shallow one due to increase curvature (Figure 11). In order to examine the wind loading on the dish structure, the drag coefficients were determined. By comparing the drag coefficient of the three dishes, it was found that the maximum drag coefficient, as expected, 
was observed in deep dish structure (Figure 12). As expected, by comparing the aerodynamic loading on the selected three different dishes, the maximum drag coefficient was observed in deep dish structure. The observation showed that an increase in the curvature produces higher drag coefficient on the structure. Also, the important point to note is that the forces on the dish structure increased by increasing the depth of the dish structure for all the positive tilt angles, and up to some extent with negative tilt angle. But surprisingly, between the range of $-45^{\circ}$ and $-90^{\circ}$ tilt angle, the drag coefficient experienced by the dish has been observed lesser than the other two dishes.

\subsection{Convective heat losses}

Based on the observations discussed above, the difference was found in the drag coefficient when comparing dishes with different focal lengths. In turn this raised some interesting questions into how the heat loss from the receiver is influenced by this change. In considering how the positioning of the cavity receiver may impact on the heat loss, Figure 13 shows the convective heat losses from the cavity receiver of all three cases.

The results show a slightly lower heat loss from the three dishes over a range of tilt angles. It is clear from the Figure 12 that deep dish provides a slightly greater sheltering effect on the receiver due to its shorter focal length.

Due to the sheltering effect of deep dish on receiver, the local air velocity near the cavity receiver was lower than the free stream air velocity. The reduction in the local air velocity prevented the heat loss from the receiver. Whereas, the heat loss from the shallow dish was observed to be maximum in all cases due to the location of the cavity receiver in the region of highest free air stream velocity.

For zero degree tilt angle, although the receiver is in the free stream region, the convective heat loss is still less than other cases due to boundary layer generated by the dish structure. 
The overall results showed a significant sheltering effect on the cavity receiver provided by the deep dish and subsequently on the convective heat loss.

\section{Conclusion}

The study provided an effect of the receiver's positioning on two important parameters that affect the system performance i.e. the wind loads and the convective heat losses from the receiver. The result indicated significant effect of the dish on the local flow near the receiver in most of the cases. Also, the maximum drag coefficient was observed in deep dish structure compared with other cases of medium and shallow dishes. However, relatively small differences in drag coefficients for all three cases imply that the aerodynamic behaviour is almost same for most of the tilt angles of the dish.

Furthermore, the observation showed the impact of the dish on the convective heat losses over a range of tilt angles. The convective heat losses from the receiver were significantly changed due to the orientation of the dish-cavity system. Despite this observation, it is important to note that the heat loss from the receiver varies more with tilt angle than it does with focal length (or dish depth). Thus it can be concluded that the heat loss is a stronger function of tilt angle rather than focal length, and in essence, the heat loss due to variation of this are negligible.

\section{Reference}

Abbasi-Shavazi, E., Torres, J.F., Hughes, G. and Pye, J., 2020, "Experimental correlation of natural convection losses from a scale-model solar cavity receiver with non-isothermal surface temperature distribution", Solar Energy, Vol 198, pp.355-375. 
Alipourtarzanagh, E., Chinnici, A., Nathan, G.J. and Dally, B.B., 2020, "Experimental insights into the mechanism of heat losses from a cylindrical solar cavity receiver equipped with an air curtain”, Solar Energy, Vol 201, pp. 314-322.

Alnaqi, A. A., Alsarraf, J., \& Al-Rashed, A. A. (2021). Numerical investigation of hydrothermal efficiency of a parabolic dish solar collector filled with oil based hybrid nanofluid. Journal of the Taiwan Institute of Chemical Engineers.

Carrillo-Berdugo, I., Estellé, P., Sani, E., Mercatelli, L., Grau-Crespo, R., Zorrilla, D., \& Navas, J., 2021, Optical and Transport Properties of Metal-Oil Nanofluids for Thermal Solar Industry: Experimental Characterization, Performance Assessment, and Molecular Dynamics Insights. ACS Sustainable Chemistry \& Engineering, 9(11), 4194-4205.

Danish, S. N., Almutairi, Z., El-Leathy, A., Al-Ansary, H., Jardan, Y., \& Alaqel, S., 2021, Modeling and Performance Simulation of an Innovative Concept of Linear Fresnel Reflector based CSP System. Journal of Thermal Science, 1-11.

Giaconia, A., Iaquaniello, G., Metwally, A. A., Caputo, G., \& Balog, I. (2020). Experimental demonstration and analysis of a CSP plant with molten salt heat transfer fluid in parabolic troughs. Solar Energy, 211, 622-632.

Li, Xuan, Xuebin Wang, Fan Li, Tao Niu, Guoqiang Lu, Yue Sun, Wei Huang, Bo Hu, and Kaigui Xie. 2021, "Wind-Thermal-CSP Bundling Model with an Adjustable Heat Storage Strategy for CSP Stations." IEEE Access 9 : 25984-25992

Loni, R., Asli-Areh, E. A., Ghobadian, B., Kasaeian, A. B., Gorjian, S., Najafi, G., \& Bellos, E., 2020, "Research and review study of solar dish concentrators with different nanofluids and different shapes of cavity receiver: Experimental tests”, Renewable Energy, Vol 145, pp. 783-804. 
Lupfert, E., Geyer, M., Schiel, W., Esteban, A., Osuna, R., and Zarza, E., 2001, "EUROTHROUGH design issues and prototype testing at PSA", Proceedings of Solar Forum 2001, Solar Energy: The Power to Choose, Washington DC.

Mehrpooya, M., Ghorbani, B., \& Sadeghzadeh, M. (2019). Hybrid solar parabolic dish power plant and high-temperature phase change material energy storage system. International Journal of Energy Research, 43(10), 5405-5420.

Mohr, M., Svoboda, P., Unger, H. 1999, "Praxis solarthermischer Kraftwerke." Berlin, Heidelberg: Springer

Paitoonsurikaran, S., and Lovegrove., K., 2003, "On the study of convection loss from open cavity receiver in solar paraboloidal dish applications" proceeding of 41 st Conference of the Australia and New Zealand Solar Energy Society (ANZSES), Melbourne, Australia.

Philibert, C., 2014, “Technology Roadmap: Solar Thermal Electricity”, International Energy Agency (IEA), OECD Publication, France.

Price H., Lupfert E., \& Kearney D., 2002, “Advances in Parabolic Trough Solar Power”, Solar Engineering, Vol 124(2), pp.109-126

Reddy, K.S., Srihari, V. T., \& Veershetty G., 2015, “Combined heat loss analysis of solar parabolic dish - modified cavity receiver for superheated steam generation", Solar Energy, Vol 121, pp. 78-93.

Reddy, K. S., Veershetty, G., \& Srihari, V. T., 2016, "Effect of wind speed and direction on convective heat losses from solar parabolic dish modified cavity receiver", Solar Energy, Vol 131, pp.183-198.

Sedighi, M., Taylor, R.A., Lake, M., Rose, A., Izadgoshasb, I. and Padilla, R.V., 2020, "Development of a novel high-temperature, pressurised, indirectly-irradiated cavity receiver”, Energy Conversion and Management, Vol 204, p.112175. 
Sasa Pavlovic, Ahmed M. Daabo, Evangelos Bellos, Velimir Stefanovic, Saad Mahmoud, Raya K. Al-Dadah, 2017, "Experimental and numerical investigation on the optical and thermal performance of solar parabolic dish and corrugated spiral cavity receiver",Journal of Cleaner Production, Vol 150, pp. 75-92,

Steinfeld, A., 2005, "Solar thermochemical production of hydrogen-a review" Solar Energy, Vol 78, pp. 603-615.

Thirunavukkarasu, V., \& Cheralathan, M., 2020, An experimental study on energy and exergy performance of a spiral tube receiver for solar parabolic dish concentrator. Energy, 192, 116635.

Tyner, C. E., Kolb, G. J., Geyer, M., \& Romero, M., 2001, “Concentrating solar power in 2001 an IEA/Solar PACES summery of present status and future prospectus", presented at the meeting of the Solar PACES, Retrieved from www.solarpaces.org (Last cited: 25-042016)

Uzair, M., Anderson, T.N., and Nates, R.J., 2017, "The impact of the parabolic dish concentrator on the wind induced heat loss from its receiver", Solar Energy, Vol. 151, pp. 95101

Uzair, M., Anderson, T.N. and Nates, R.J., 2018. Modeling of convective heat loss from a cavity receiver coupled to a dish concentrator. Solar Energy, 176, pp.496-505.

Wang, M., and Siddiqui, K., 2010, "The impact of geothermal parameters on the thermal performance of a solar receiver of dish-type concentrated solar energy system", Renewable Energy, Vol 35, pp. 2501-2513.

Wu, S.,Y., Xiao, L., Cao, Y., \& Li, Y. R.,2010, “Convection heat loss from cavity receiver in parabolic dish solar thermal power system: A review", Solar Energy, Vol. 84, 1 pp. 3421355. 
Xiao, L., Guo, F.W., Wu, S.Y. and Chen, Z.L., 2020, “A comprehensive simulation on optical and thermal performance of a cylindrical cavity receiver in a parabolic dish collector system”, Renewable Energy, Vol 145, pp.878-892.

Yang, J., Yang, Z., \& Duan, Y., 2021, Load matching and techno-economic analysis of CSP plant with S-CO2 Brayton cycle in CSP-PV-wind hybrid system. Energy, 223, 120016.

Yuan, Y., Xiaojie, L., Ziming, C., Fuqiang, W., Yong, S. and Heping, T., 2020, "Experimental investigation of thermal performance enhancement of cavity receiver with bottom surface interior convex”, Applied Thermal Engineering, Vol 168, pp.114847. 


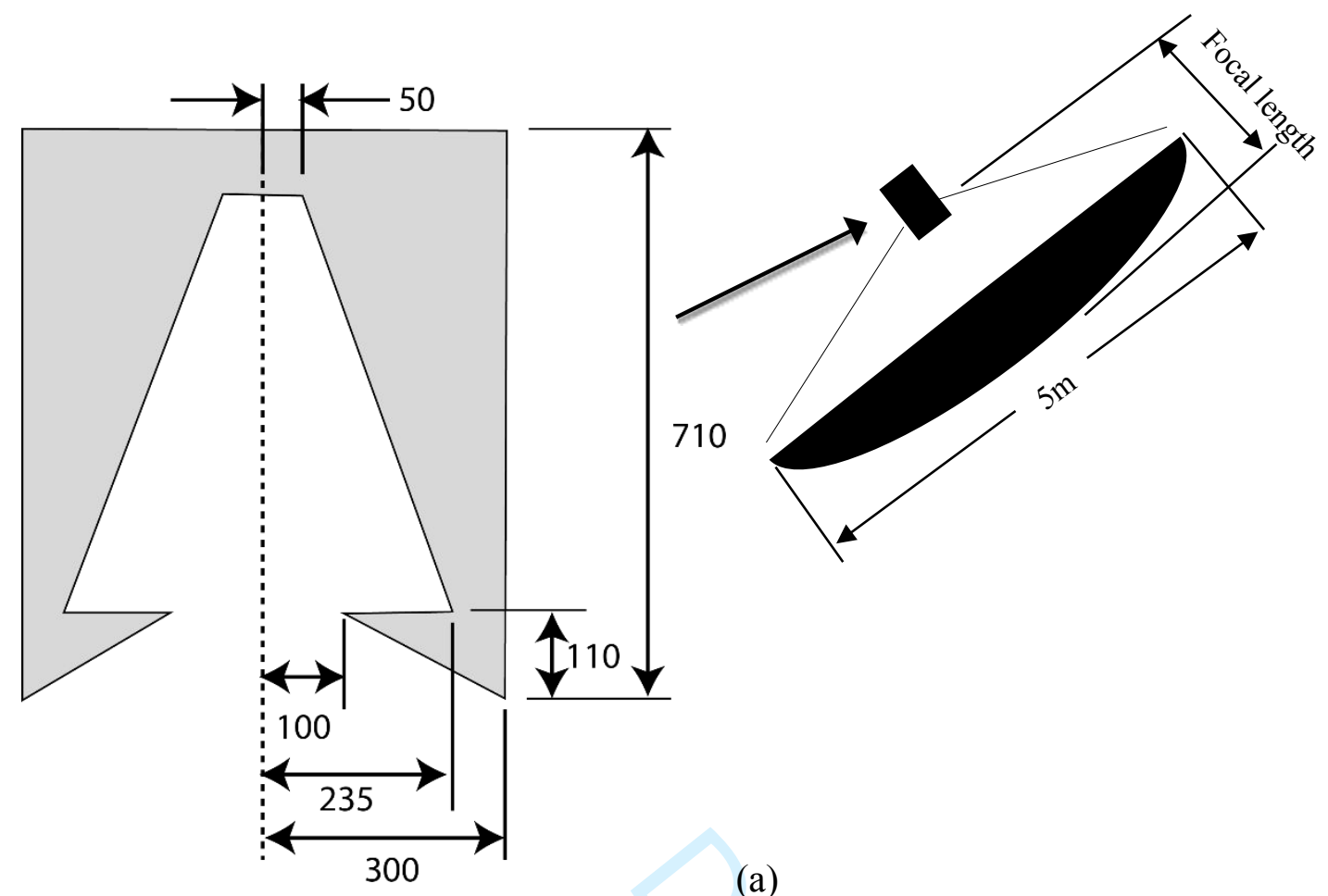

(a)

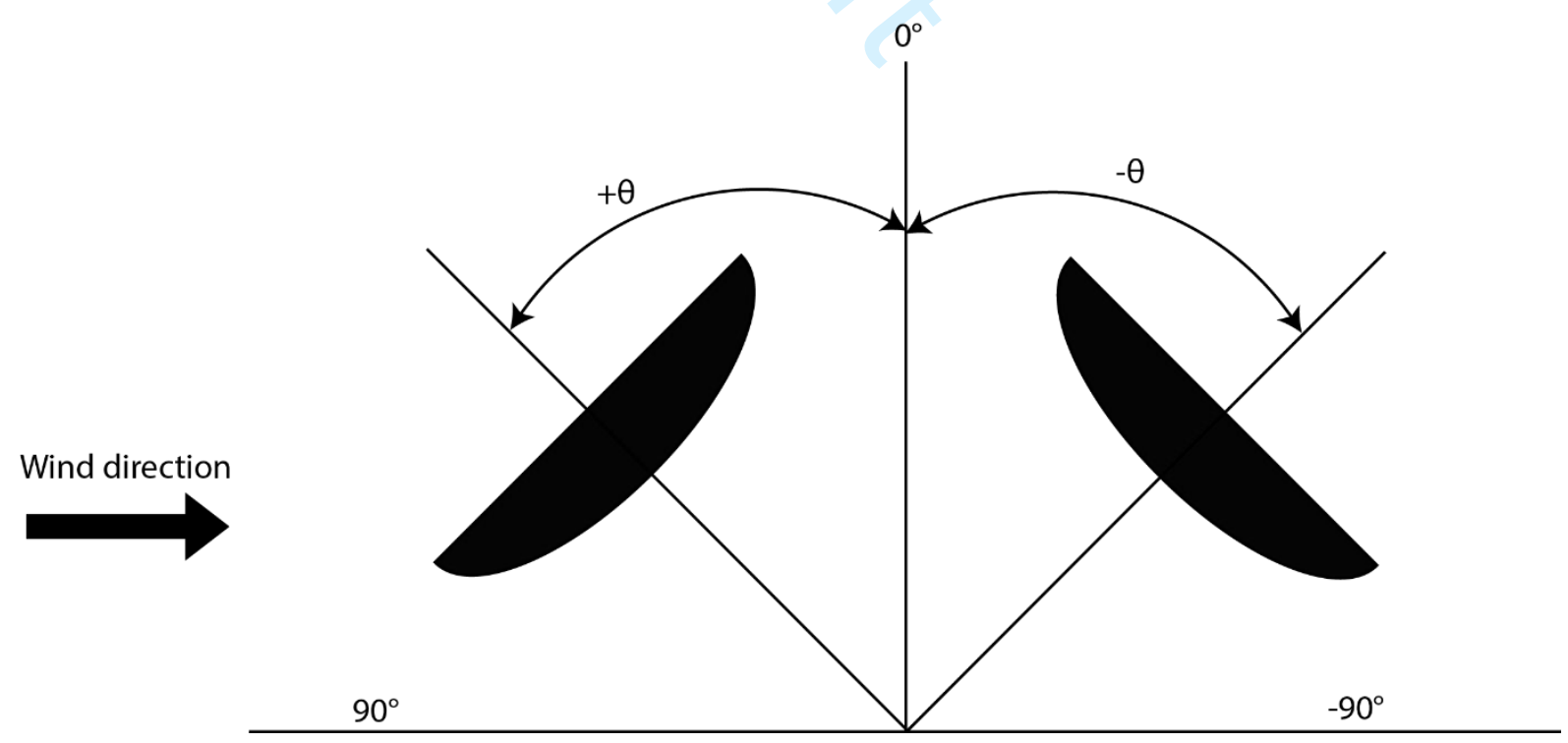

(b)

Figure 1: (a) Schematic of parabolic dish system (b) Orientation of dish with respect to different tilt angles 

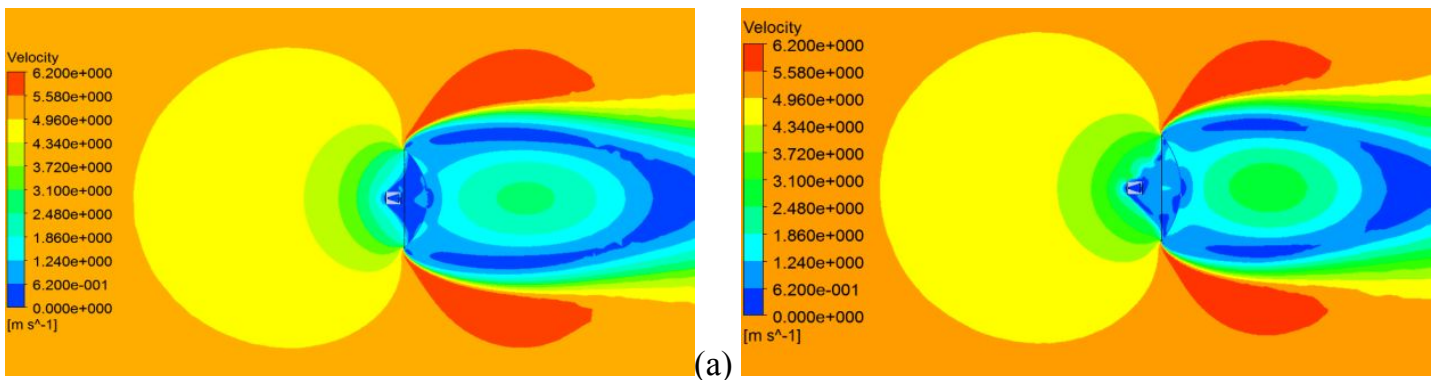

(b)

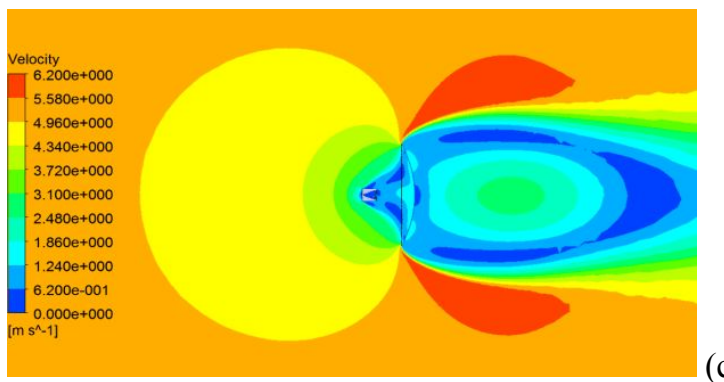

(c)

Figure 2: Velocity profile at $90^{\circ}$ tilt angle with dish having the focal lengths of (a) $1.25 \mathrm{~m}$ (b) $1.84 \mathrm{~m}$ (c) $2.5 \mathrm{~m}$ 


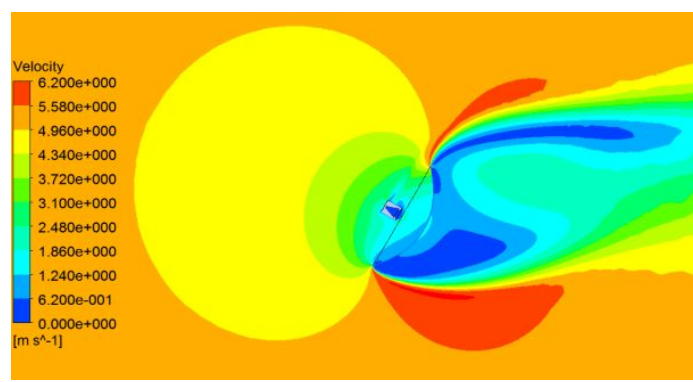

(a)

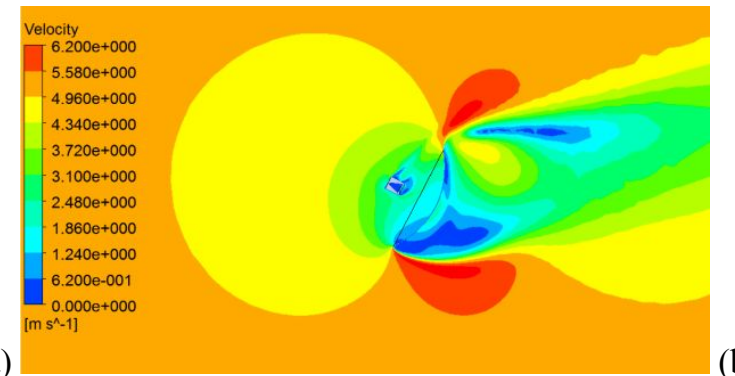

(b)

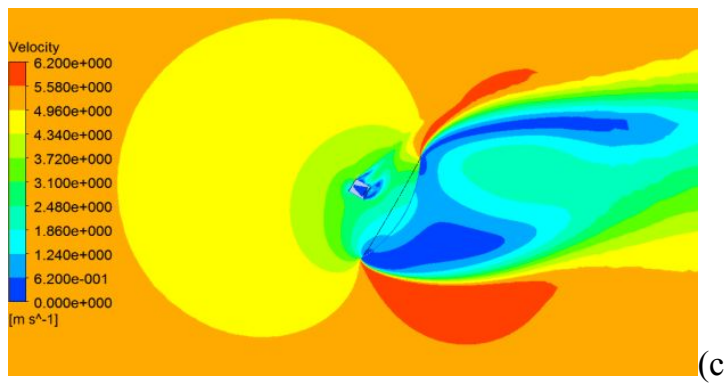

Figure3: Velocity profile at $60^{\circ}$ tilt angle with dish having the focal lengths of (a) $1.25 \mathrm{~m}$ (b) $1.84 \mathrm{~m}$ (c) $2.5 \mathrm{~m}$ 

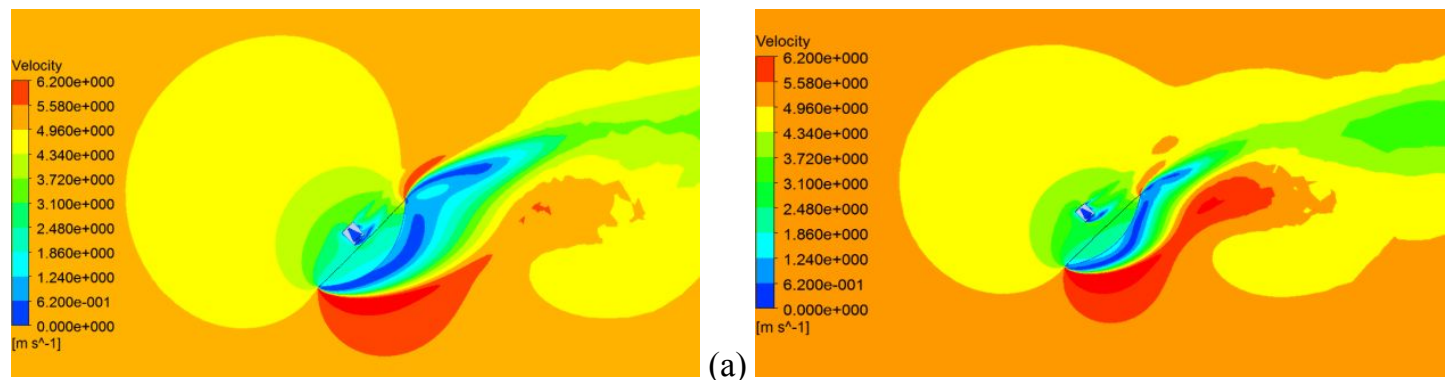

(b)

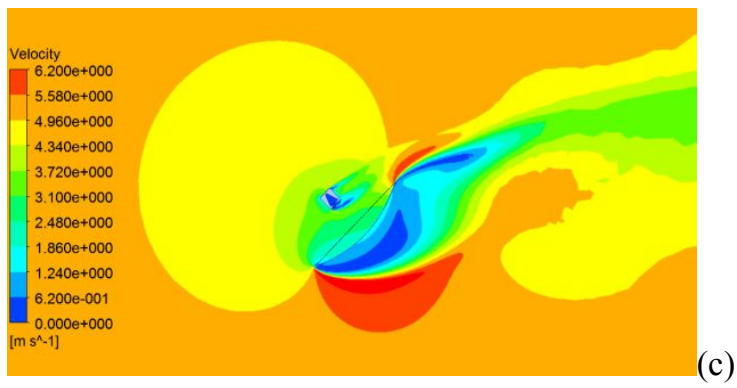

Figure 4: Velocity profile at $45^{\circ}$ tilt angle with dish having the focal lengths of (a) $1.25 m$ (b) $1.84 m$ (c) $2.5 m$ 

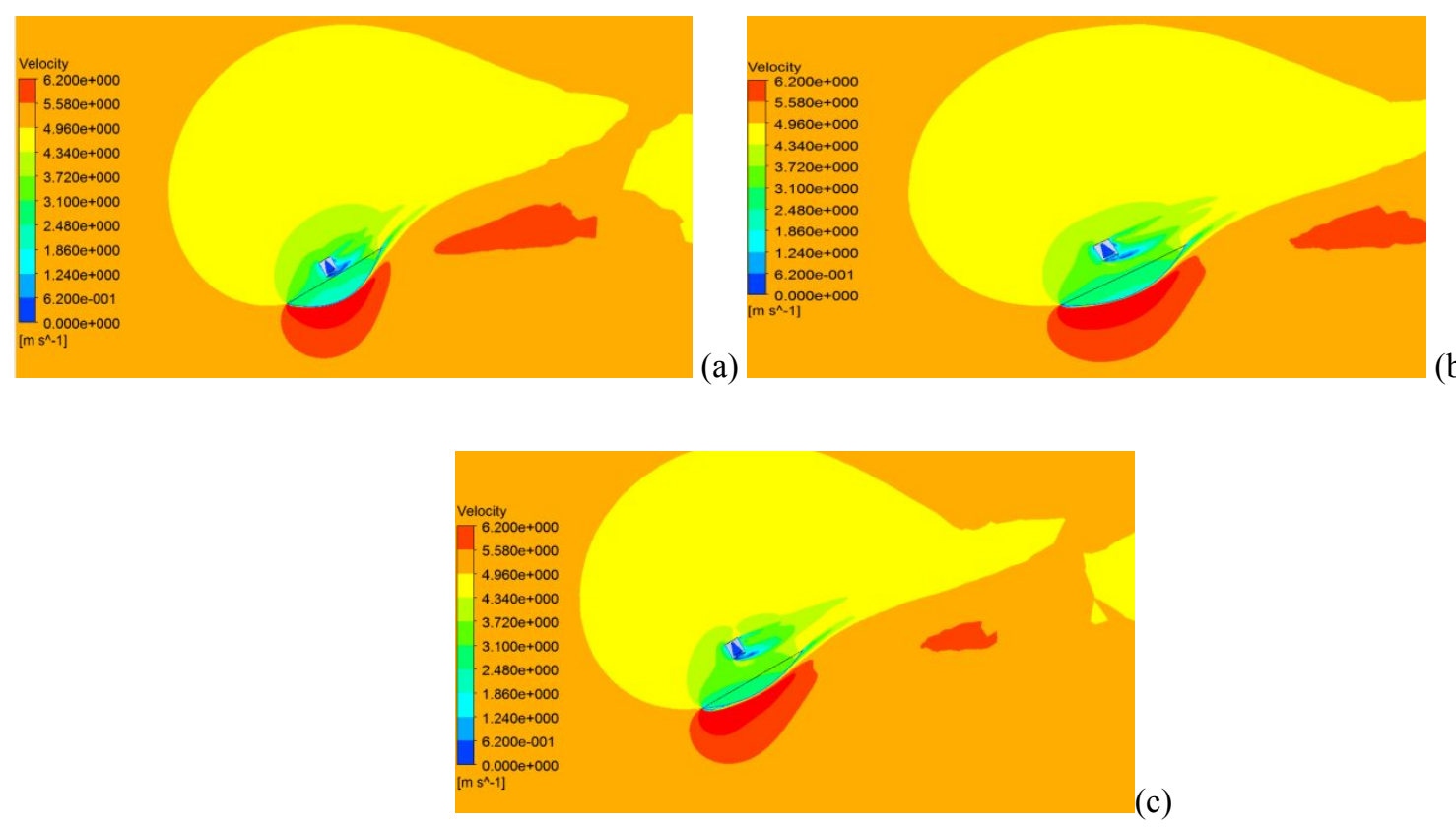

Figure 5: Velocity profile at $30^{\circ}$ tilt angle with dish having the focal lengths of (a) $1.25 \mathrm{~m}$ (b) $1.84 \mathrm{~m}$ (c) $2.5 \mathrm{~m}$ 

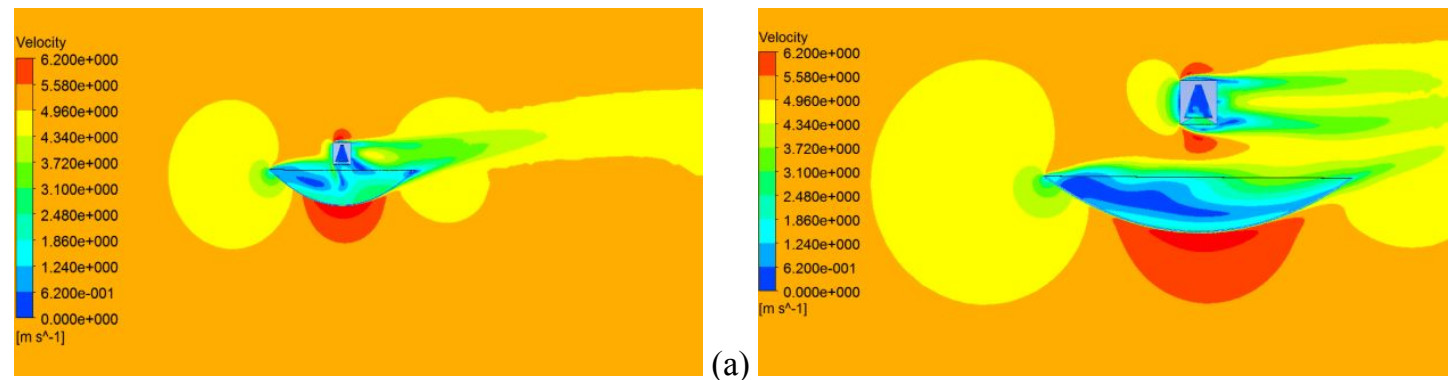

(b)

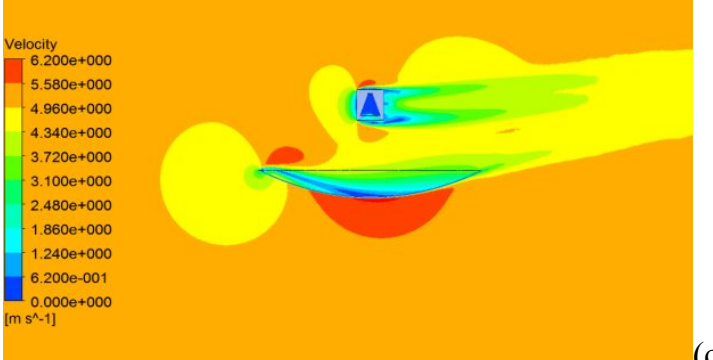

Figure 6: Velocity profile at $0^{\circ}$ tilt angle with dish having the focal lengths of (a) $1.25 m$ (b) $1.84 m$ (c) $2.5 m$ 

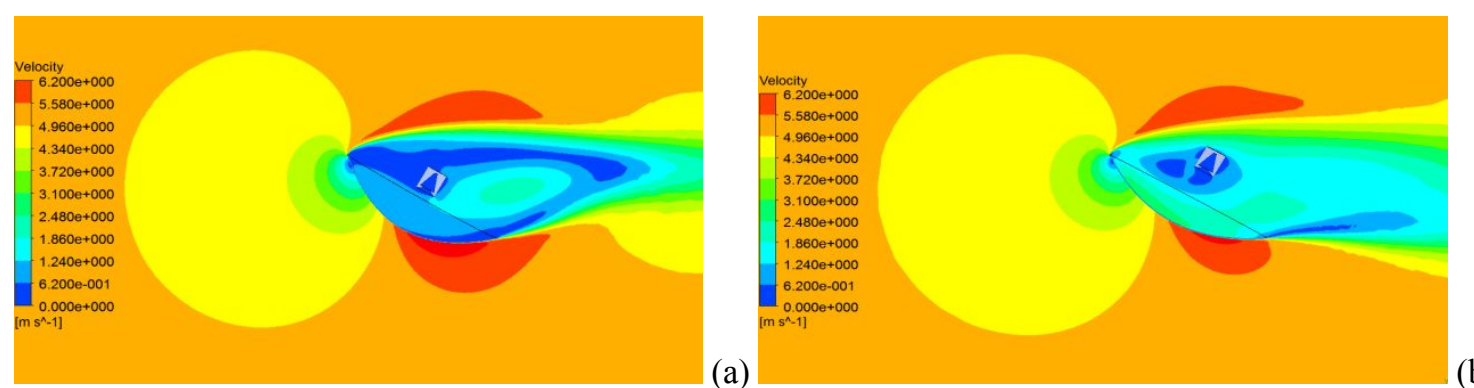

(b)

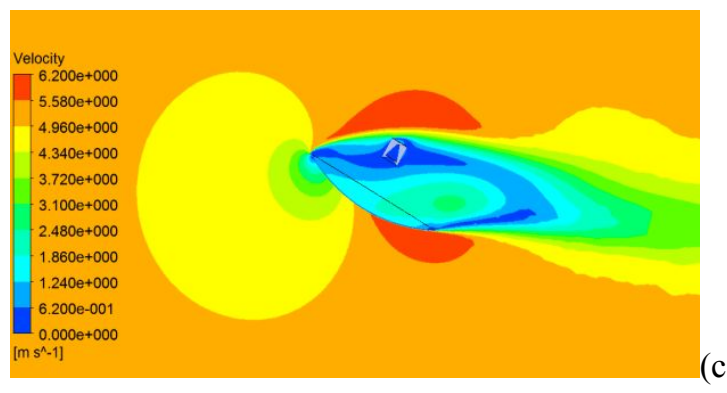

Figure 7: Velocity profile at $-30^{\circ}$ tilt angle with dish having the focal lengths of (a) $1.25 \mathrm{~m}$ (b) $1.84 \mathrm{~m}$ (c) $2.5 \mathrm{~m}$ 

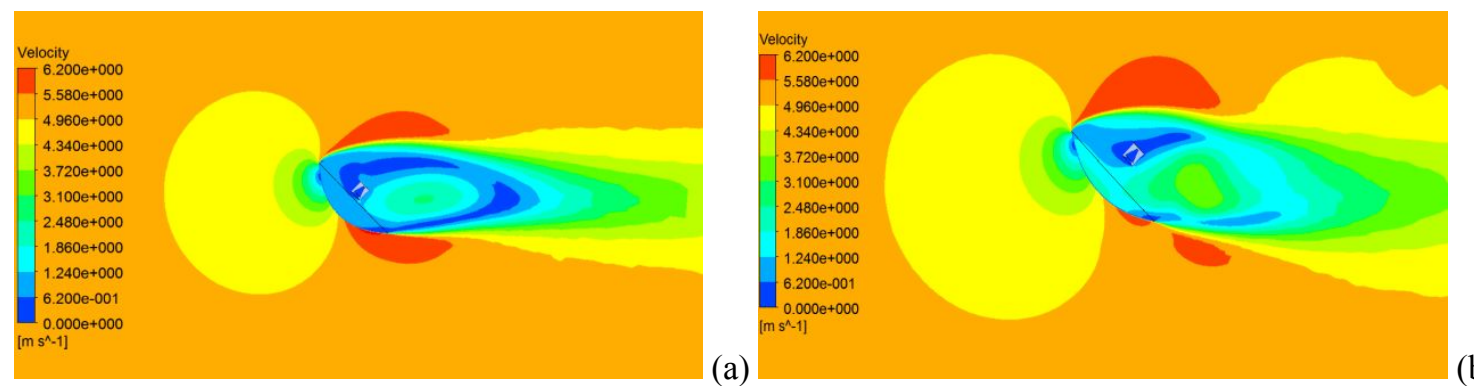

(b)

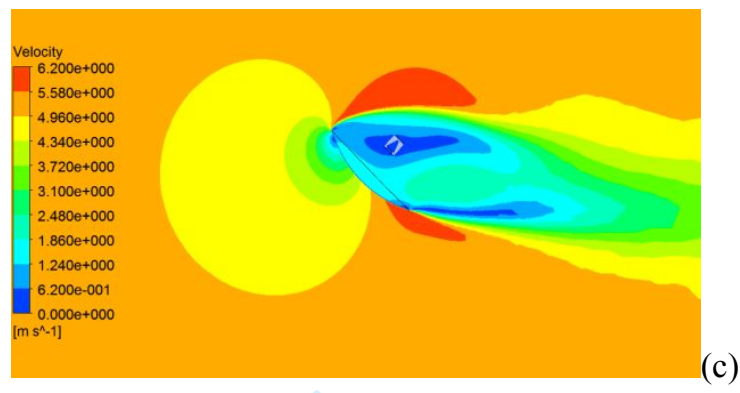

Figure 8: Velocity profile at $-45^{\circ}$ tilt angle with dish having the focal lengths of (a) $1.25 m$ (b) $1.84 m$ (c) $2.5 m$ 

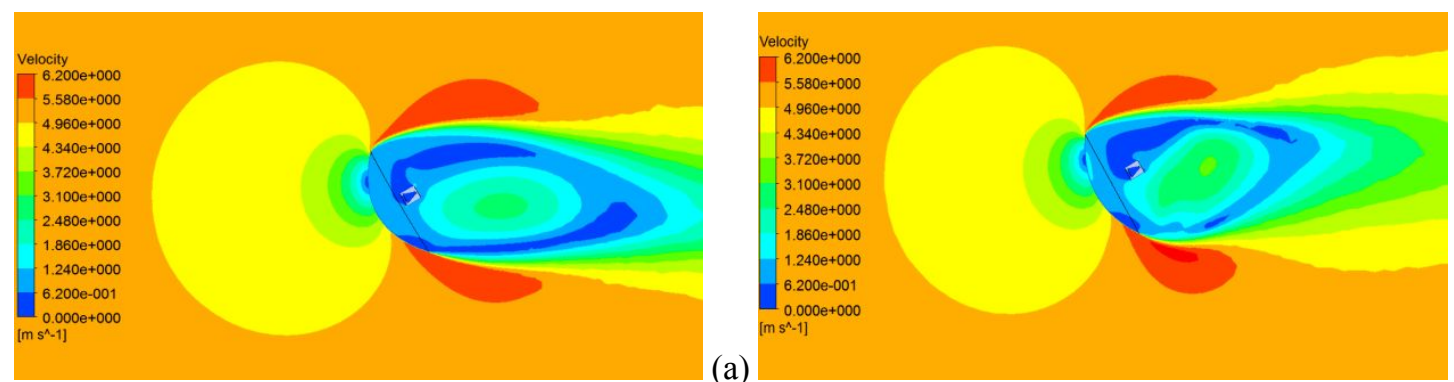

(b)

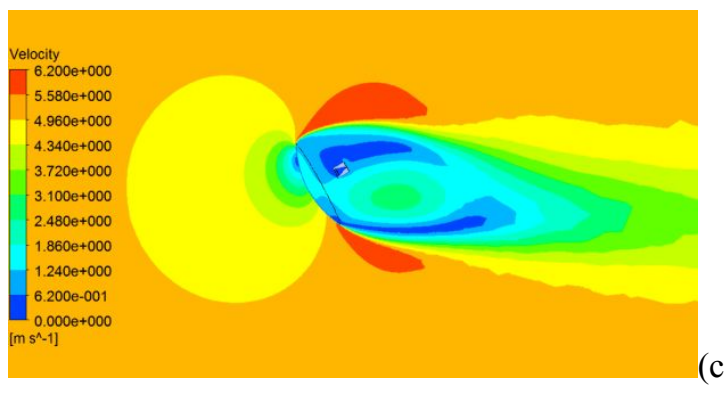

Figure 9: Velocity profile at $-60^{\circ}$ tilt angle with dish having the focal lengths of (a) $1.25 m$ (b) $1.84 m$ (c) $2.5 m$ 

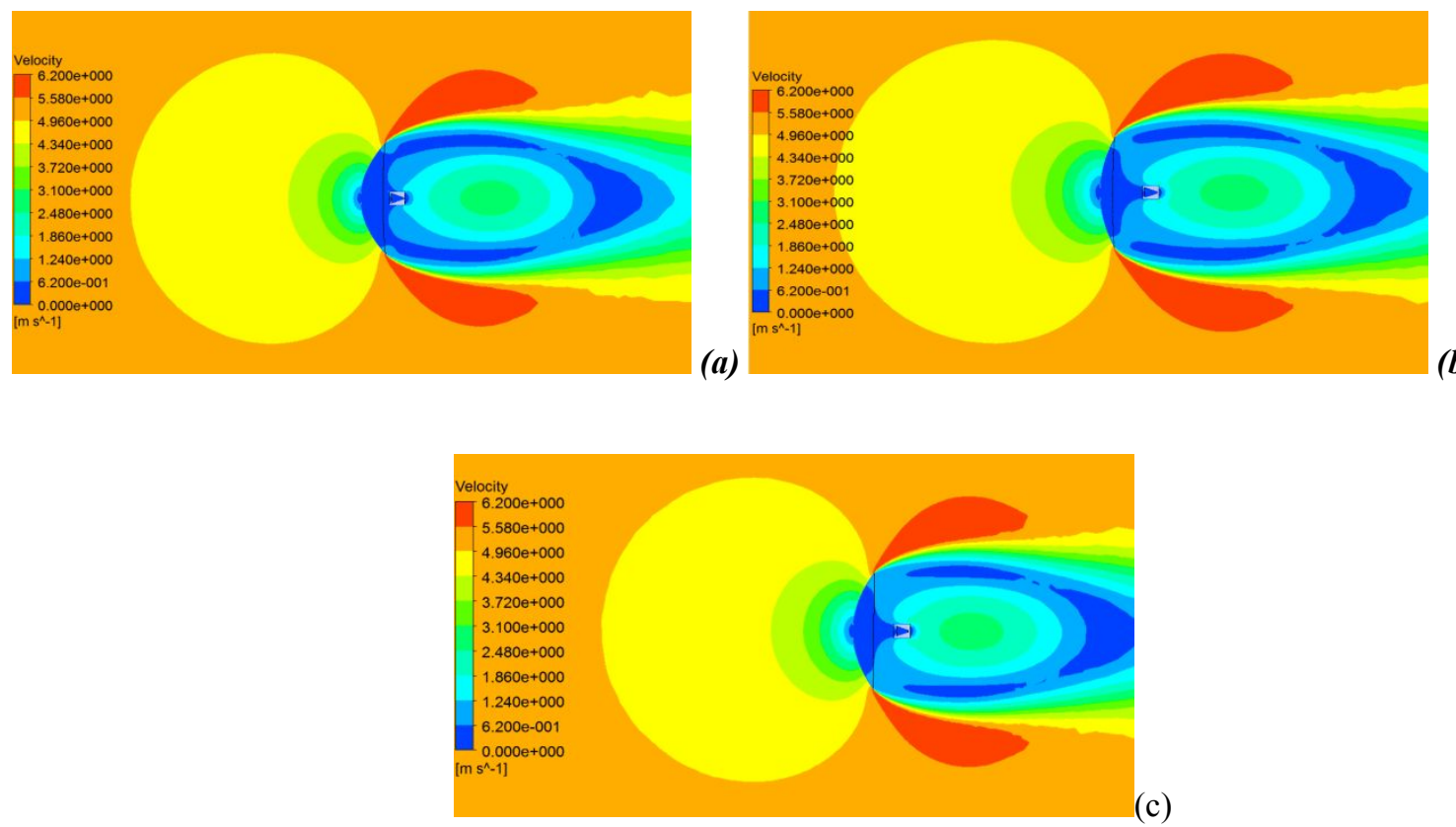

Figure 10: Velocity profile at $-90^{\circ}$ tilt angle with dish having the focal lengths of (a) $1.25 \mathrm{~m}$ (b) $1.84 \mathrm{~m}$ (c) $2.5 \mathrm{~m}$ 


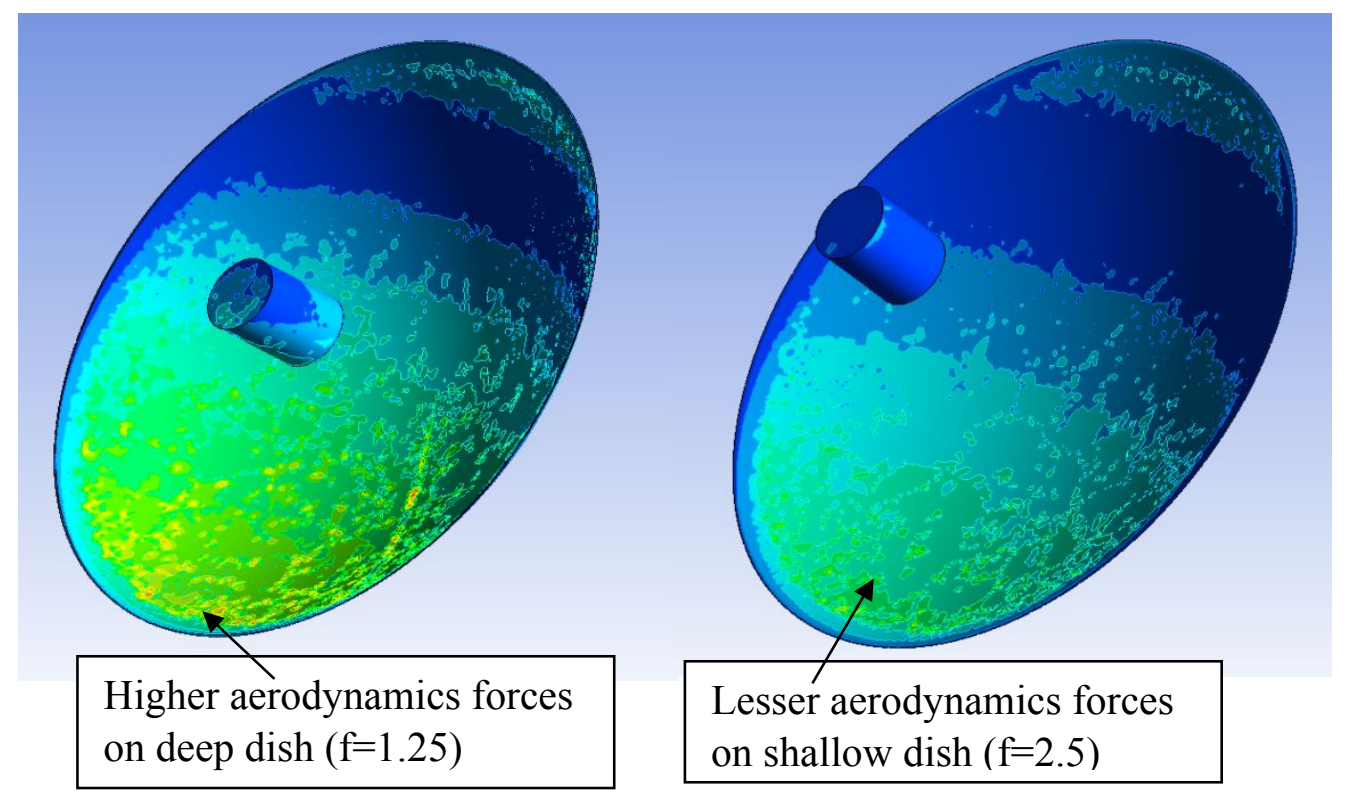

Figure 11: Comparison of aerodynamic forces on the dish 


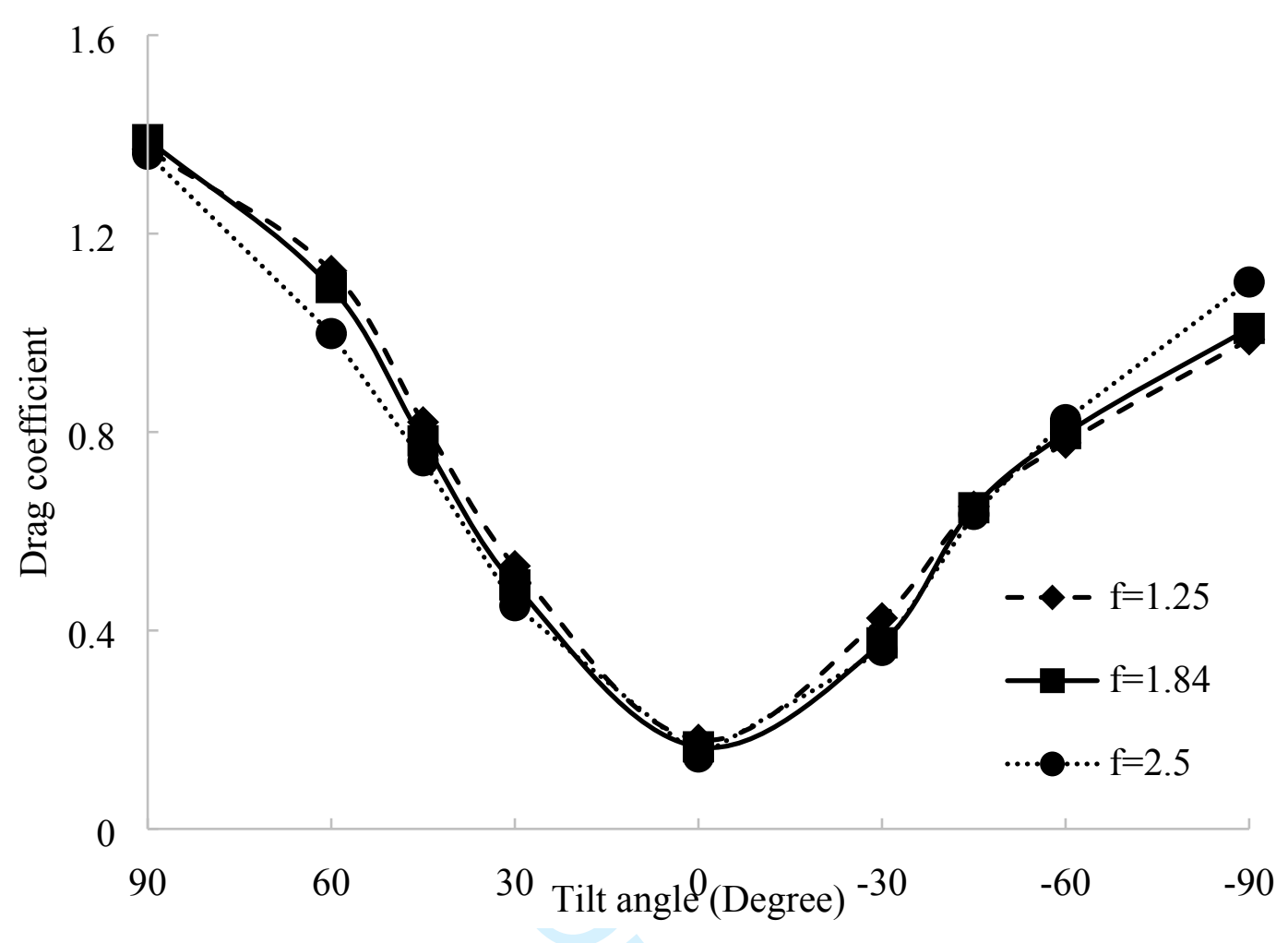

Figure 12: Drag coefficients at different tilt angles for the selected dish geometries 


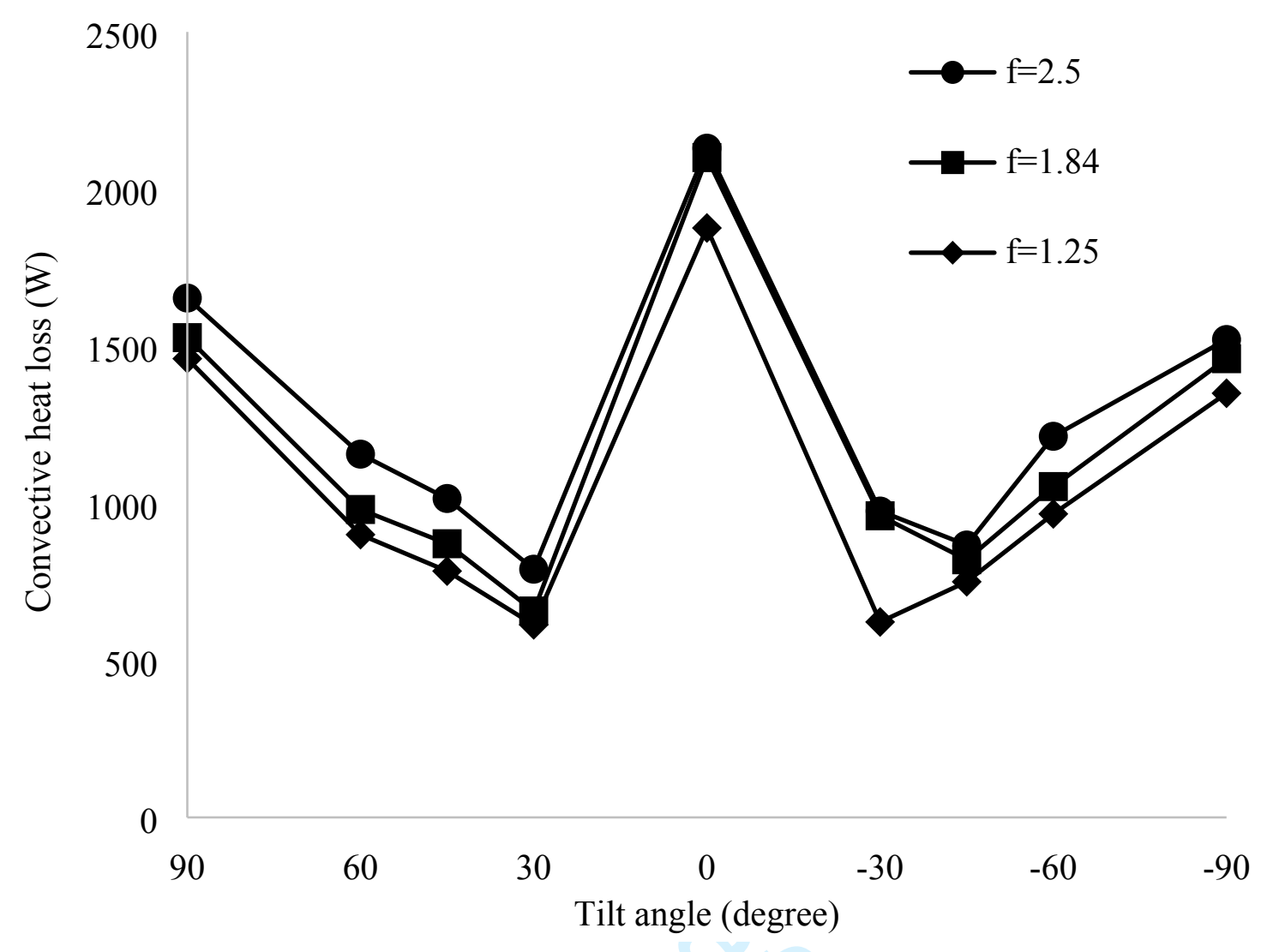

Figure 13: Convective heat loss from the cavity receiver with different focal lengths 